\title{
DETECTION AND CHARACTERIZATION OF CHEMICAL AND BIOLOGICAL AEROSOLS USING LASER-TRAPPING SINGLE-PARTICLE RAMAN SPECTROSCOPY
}

\author{
AIMABLE KALUME ${ }^{1}$, ZHIYONG GONG $^{2}$, CHUJI WANG ${ }^{2}$, JOSHUA SANTARPIA ${ }^{3}$ \& YONG-LE PAN ${ }^{1}$ \\ ${ }^{1}$ U.S. Army Research Laboratory, Adelphi, USA \\ ${ }^{2}$ Mississippi State University, Starkville, USA \\ ${ }^{3}$ Sandia National Laboratories, Albuquerque, USA
}

\begin{abstract}
Detection and characterization of the presence of chemical and biological agent aerosols in various complex atmospheric environments is essential. Raman spectroscopy has the ability to identify chemical molecules, but there are a limited number of photons detectable from each single airborne aerosol particle as it flows through a detecting system. Here, we report a single-particle Raman spectrometer that can measure strong spontaneous, stimulated, and resonance Raman spectra from a single laser-trapped airborne aerosol particle. Samples such as the VX nerve agent chemical simulant diethyl phthalate and various pollens were tested. A particular optical trap using two focusing counterpropagating hollow beams was able to stably trap both absorbing and non-absorbing particles in air for long observation times. Using this system, time-resolved Raman spectra and elastic scattered intensities were recorded to monitor the chemical properties and size variation of the trapped particle. Specifically, the developed technology was able to measure the Raman spectra from different submicron positions within a single laser-trapped airborne particle. Such a system provides a new approach for the detection and characterization of single airborne chemical- and biological-agent aerosol particles, as well as.to detect and monitor the molecules and molecular reactions located in different positions within a micronsized particle as it responds to various micro-local environments.
\end{abstract}

Keywords: chemical and biological agent aerosols, submicron position-resolved raman spectra, laser trapping, single airborne aerosol particle.

\section{INTRODUCTION}

Airborne aerosol particles play a very important role in the atmosphere, where their physical, chemical, and biological properties, as well as their interactions with the multifaceted environments have significant impacts on earth climate and human health. Atmospheric aerosol particles are composed of organic and/or inorganic constituents, in solid, liquid, aqueous, or colloid form. Extensive research has studied the properties of these aerosol particles, either from particle ensembles or extracts of particles collected onto substrates, using a variety of observing techniques. Typically, each method provides a partial view into the extremely complex nature of their interaction with the atmosphere. In order to have a better understanding of the particles' morphology, chemical composition, phase formation, nucleation etc., researchers have advanced the technologies to characterize aerosols based on the measurements of individual single particles either from samples collected on a substrate or in solution; from individual particles flowing-through a sensor; or from stably trapped single particles. The combination of particle trapping with laser spectroscopic characterization enables the monitoring of the particle's time-resolved interactions and reactions under various controlled atmospheric conditions. In addition, the reported approach helps reduce the interference from extraneous materials, e.g. scattering and stray light from a supporting substrate. It also allows the particle's entire surface to freely interact with its surrounding environment as it does in its natural state in the atmosphere. 
Raman spectroscopic technologies have been widely used in material characterization in chemistry, physics, biology, aerosol science, and life science [1]-[4]. Laser trapping with Raman spectroscopy (LTRS) enables studying a single micro-size particle, spore or cell, then monitoring the temporal process of a particle under various environments. It has been extensively used in the study of molecular biology with the help of microscopy. However, a biological system even a single cell is a complex mixture containing numerous biomolecules in various concentrations and forms, including amino acids, proteins, nucleic acids, coenzymes, flavins, lipids, and more. Some cells or spores can grow, change, and reproduce in buffer liquid or air. However, different molecules located in different areas within a particle have different functions and responses to various environments. In order to detect and monitor the different biomolecules as they respond to the changes of various environments, then gain new fundamental understanding of these reactions in a particle or a cell, measuring the Raman spectra from the different positions within a single cell, or a particle could be a very powerful tool. The reported position-resolved Raman spectra are realized either by recording multiple Raman spectra via spatially scanning the sample, or measuring multiple monochromatic images via scanning a spectral filter to collect the scattering signal onto a 2-D detector at multiple wavelengths. However, these results are traditionally obtained either from samples on a substrate or from bulk samples. Although there are numerous studies using the LTRS technique, the majority of them have been performed in a liquid solution, with an increasing number of studies conducted in air [1]-[4], there have been no study that could record the position-resolved Raman spectra from laser-trapped single airborne aerosol particles. Here, we demonstrated the measurement of the position-resolved Raman spectra within a lasertrapped single airborne particles.

\section{EXPERIMENTAL ARRANGEMENT}

As shown in Fig. 1, the $488 \mathrm{~nm}$ laser beam, which was used for particle trapping, was also used for Raman scattering excitation. Raman scattering and elastic scattering signals from individual single trapped particle were collected with a microscope objective lens (Mitutoyo $20 \times, \mathrm{NA}=0.42$ ), then the collimated scattering light was separated, the elastic scattering at $488 \mathrm{~nm}$ was reflected by a dichroic beam splitter (long pass, cutoff wavelength $488.5 \mathrm{~nm}$ ) and directed onto a photomultiplier tube (PMT).

The PMT recorded the elastic scattering light within the scattering angle $90^{\circ} \pm 2^{\circ}$ and was later compared with a simulated resonance scattering spectrum computed using the LorenzMie theory for particle size determination. The Raman scattering light was focused by a second lens into an image-preserving spectrograph (Princeton Instr. Isoplane SCT320). A long-pass filter was used to further block the residue of the elastically scattered $488 \mathrm{~nm}$ light. The dispersed imaging Raman spectra were recorded by an electron-multiplying chargecoupled device (EMCCD, Princeton Instr., ProEM) operating in its image mode. In general, the EMCCD operates in spectral mode for recording spectra by binning the pixels vertically.

Here, the entrance slit of the spectrograph was set to be as narrow as $10 \mu \mathrm{m}$, and then only a vertical slice of the magnified image from a single trapped particle with a width of $200 \mathrm{~nm}$ of the particle is imaged onto an electron-multiplying charge-coupled device (EMCCD). In such a condition, the image recorded on each pixel of the EMCCD corresponded to a 200 $\mathrm{nm} \times 200 \mathrm{~nm}$ area from the particle. After spectral dispersion, the vertical pixels of the EMCCD represent the vertical spatial positions of the particle and the horizontal pixels correspond to the shifted wavenumber of the Raman spectra. By horizontally scanning the imaging lens L3 step by step, each vertical strip of the particle's image enters the slit, then the 2-dimensional Raman spectra for the focused layer of the whole droplet could be obtained. 


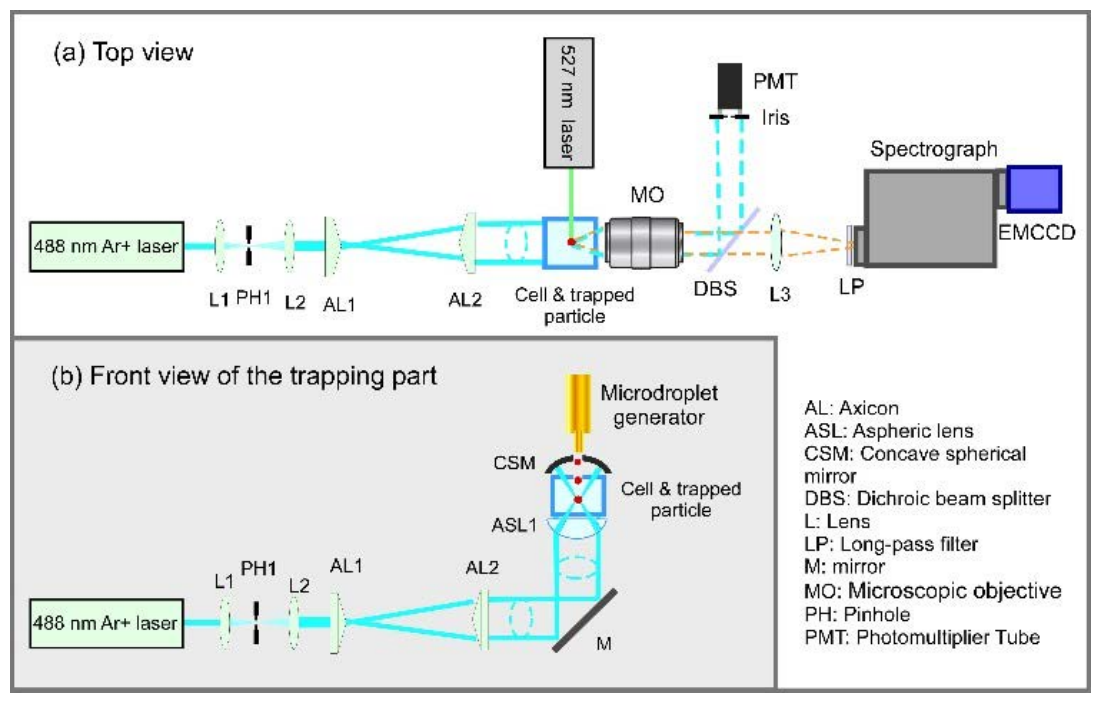

Figure 1: (a) Top view of the experimental schematic for measuring position-resolved Raman spectra of a laser-trapped single airborne aerosol particle; (b) The front view of the aerosol generator and the particle trapping part of the setup.

This arrangement acts like a confocal microscope with a narrow slit instead of a pin hole on the imaging plane.

Therefore, the count numbers from each pixel of individual horizontal lines of the EMCCD gives the Raman spectral intensity from the corresponding position of a $200 \mathrm{~nm} \times$ $200 \mathrm{~nm}$ area of the droplet at the focused depth. Once a target area (a vertical slice at a certain horizontal position at a centred layer) is focused onto the slit of the spectrograph, the Raman spectra from this vertical slice were recorded at a frame rate of $1 / 15 \mathrm{~Hz}$, each image frame is integrated during $1 \mathrm{sec}$ (of the 15) for signal accumulation. Following this procedure, the temporal profiles of a set of Raman spectra are obtained.

\section{RESULTS AND DISCUSSION}

Fig. 2 shows (a) the imaging Raman spectra of an vertically-oriented central slice of a lasertrapped diethylphthalate microdroplet $20 \mu \mathrm{m}$ in diameter, when the slit of the spectrograph was set at $10 \mu \mathrm{m}$; and (b) the position-resolved Raman spectra at 5 representative locations (each from a $200 \mathrm{~nm} \times 200 \mathrm{~nm}$ area) from a vertical cross section passing through the center of a single trapped diethyl phthalate droplet. The Raman spectra from the areas nearest the droplet surface (A and E) showed additional stimulated Raman scattering (SRS) features and whispering-gallery-modes (WGMs) resonance peaks; no such features were found in the Raman spectra measured from the interior area of the droplet (B, C, and D). Instead the spectra measured from the interior area agreed with spectral measurements from bulk liquid that contained only spontaneous scattering. While the spontaneous Raman signal is generally weak, the SRS peaks exhibit stronger and sharper profiles as evidenced by the peaks at $\Delta v=231,363,387,540,568,588$ and $1054 \mathrm{~cm}^{-1}$. The observed cavity-enhanced phenomenon has been widely reported in a droplet or microsphere, where spontaneous Stokes photons injected into the medium lead to the amplification of the Raman transitions by total internal 


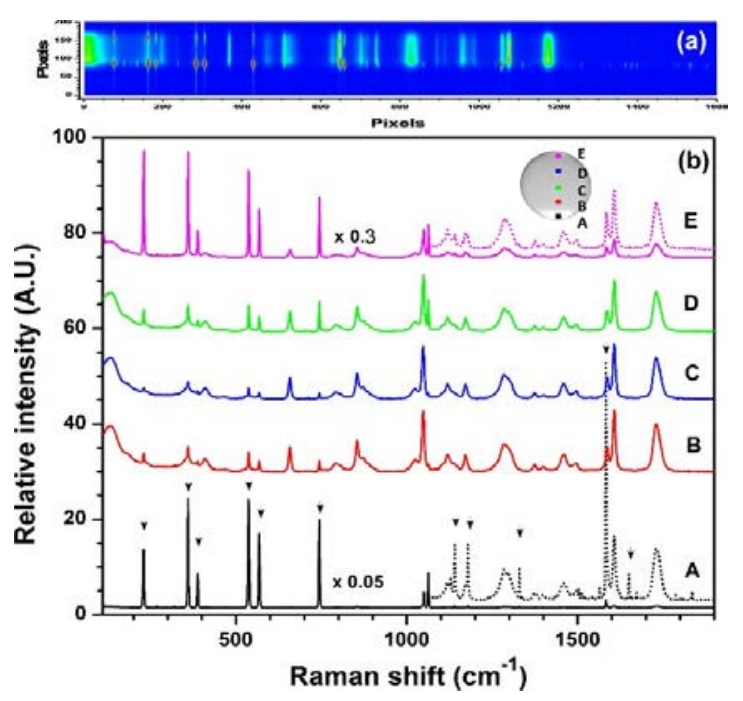

Figure 2: The imaging Raman spectra. (a) One $200 \mathrm{~nm}$ vertically-oriented central slice of a laser-trapped diethyl phthalate microdroplet with $20 \mu \mathrm{m}$ diameter; (b) The Raman spectra from the five positions marked as A, B, C, D, and E (each with an equal $200 \mathrm{~nm} \times 200 \mathrm{~nm}$ area) along the central slice vertically from the center at $-8 \mu \mathrm{m} ;-4 \mu \mathrm{m}, 0 \mu \mathrm{m} ;+4 \mu \mathrm{m}$, and $+8 \mu \mathrm{m}$ respectively.

reflection within the microcavity [5-6], this phenomena happens in Raman scattering, elastic scattering, absorption, and fluorescence. Double resonance from input illumination and output scattering resonance wavelengths accounts for very strong WGMs peaks and supplies more opportunities to produce SRS and Raman laser emissions. Thus, the WGMs related waves escape from the microcavity in the evanescent wave format tangentially, and the relative intensities of these cavity-enhanced signals depend on both the originating position in the microcavity and the angle of observation.
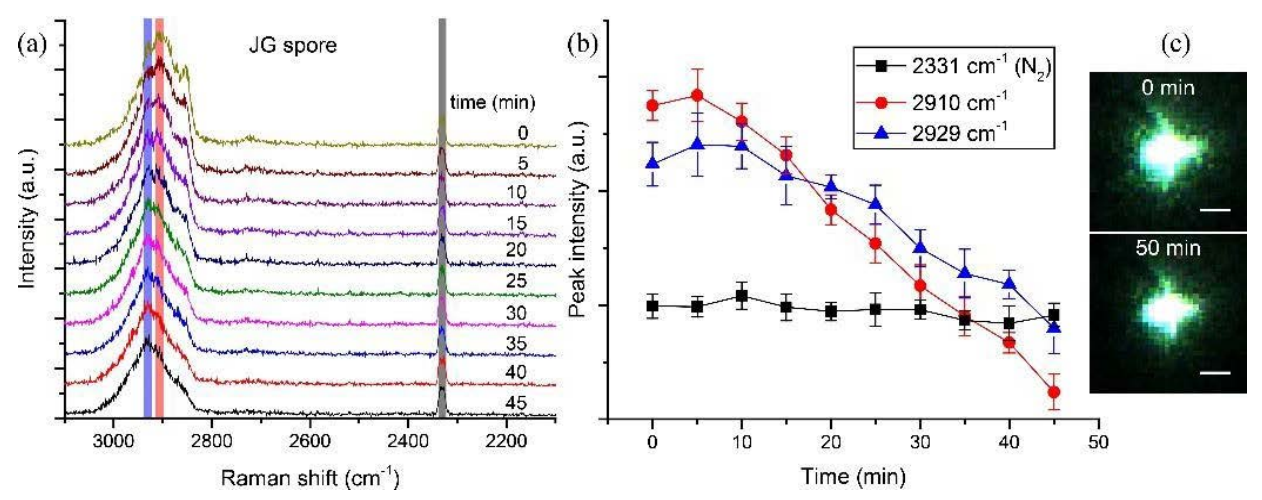

Figure 3: Temporal Raman spectra (a) of a laser-trapped Johnson grass spore (b) its intensity variation and (c) representative images. 
Fig. 3(a) shows the time-dependent Raman spectra from a single trapped bioaerosol, Johnson grass smut spore. The spectra were acquired with a slit width of $200 \mu \mathrm{m}$ with the camera set in a spectral mode (no vertical spatial resolution recorded here). The timedependent spectra were acquired consecutively with an integration time of $300 \mathrm{~s}(5 \mathrm{~min})$ for each spectrum. The profile of C-H stretching bands can be visually distinguished using the 10 frames of the Raman spectra. The quantitative examination of three peaks $\left(2331 \mathrm{~cm}^{-1}\right.$, black squares; $2910 \mathrm{~cm}^{-1}$, red dots; $2929 \mathrm{~cm}^{-1}$, blue triangles) is shown in Fig. 3(b). Obviously, the intensity of the nitrogen peak (from air) remains constant, while the C-H stretching peak at $2910 \mathrm{~cm}^{-1}$ decreased significantly faster than the peak at $2929 \mathrm{~cm}^{-1}$ and their relative intensities were reversed at 15-20 min. The images in Fig. 3(c) shows the scattering image of the trapped particle showing the reduction of intensity of emission. This temporal result from the laser trapped single bioaerosol demonstrated the system's capability of characterizing and monitoring of a single optically trapped bioaerosol particles regardless of the strongly fluorescent nature.
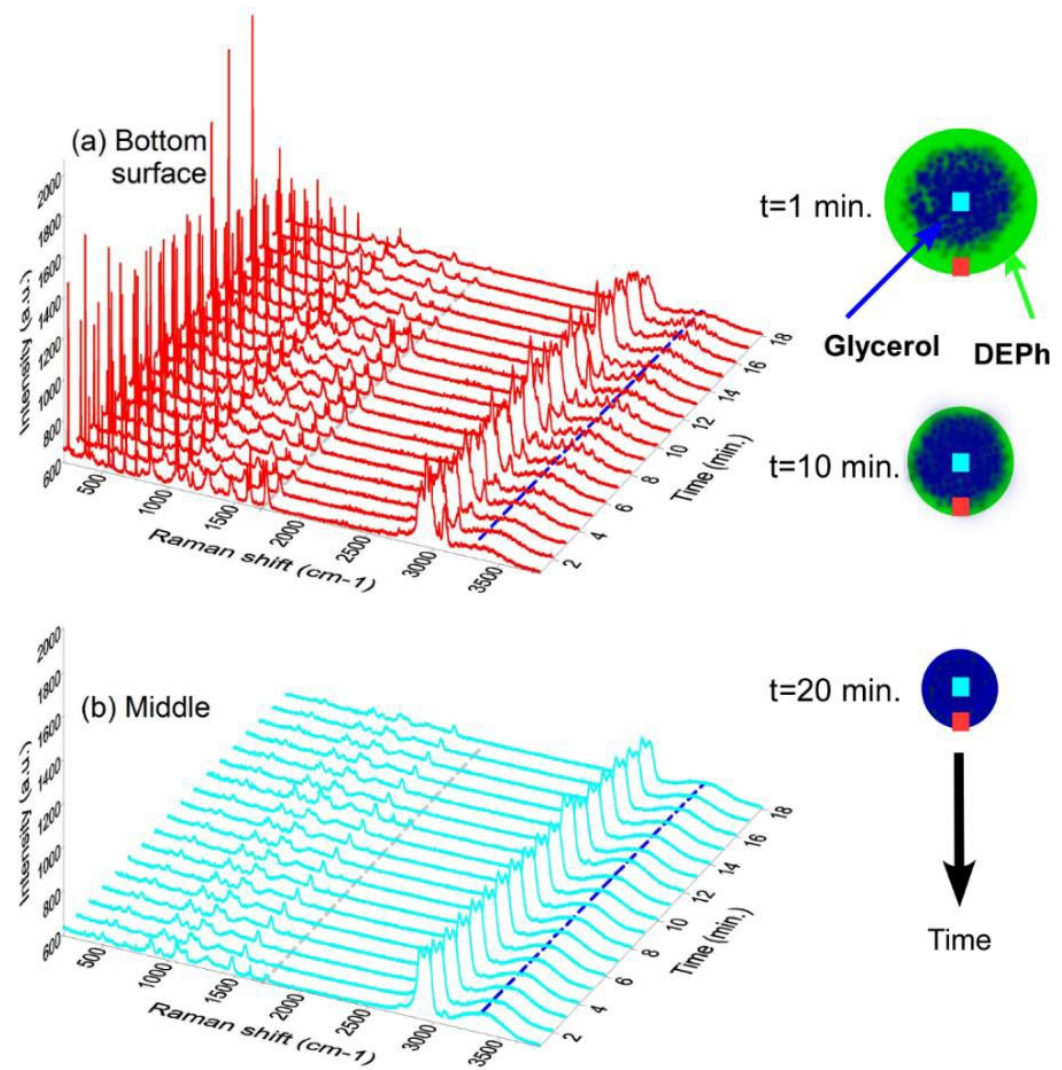

Figure 4: Typical time- and position-resolved Raman spectra from a laser-trapped droplet of mixed DEPh and glycerol. The time-resolved Raman spectra from a spot (200 $\mathrm{nm} \times 200 \mathrm{~nm}$ ) (a) near the bottom surface and (b) the center of the droplet. $\mathrm{C}=\mathrm{O}$ stretch at $\sim 1733 \mathrm{~cm}^{-1}$ and $\mathrm{O}-\mathrm{H}$ stretch band around $3366 \mathrm{~cm}^{-1}$ are the representative Raman peaks for DEPh and glycerol respectively. 
The temporal position-resolved Raman spectra can reveal the detailed morphology, liquidliquid separation and evaporation of a mixed droplet. The time-resolved Raman spectra from two representative regions, one at the bottom surface and a second at the center of a mixed droplet are shown in Fig. 4(a) and (b), respectively. The peaks (the $\mathrm{C}=\mathrm{O}$ stretch at $\sim 1733$ $\mathrm{cm}^{-1}$ and C-H stretch at $\sim 3080 \mathrm{~cm}^{-1}$ ) attributed to DEPh are primarily observed near the surface and gradually decay with time. These peaks were rarely observed within the central area. While the broad band $3366 \mathrm{~cm}^{-1}$ attributed to the $\mathrm{O}-\mathrm{H}$ stretching of glycerol is observed at both positions with a much stronger intensity (higher percentage) in the central spot (inside of the droplet) than the surface region. Notably, the intensity of the unique DEPh peak at $1733 \mathrm{~cm}^{-1}$ for both spots is barely observable after $18 \mathrm{~min}$. indicating that DEPh in the droplet has almost totally evaporated. By contrast, the intensity of the glycerol peak at $3366 \mathrm{~cm}^{-1}$ had little change at either position during this time period. This suggested that the mixed organicorganic droplet formed a core-shell structure with the glycerol primarily concentrated in the core, and the $\mathrm{DEPh}$ was predominant around the outer layer of the droplet. The strong SRS and WGM peaks were only observed at the spots around the surface region. Initially, a wellmixed solution of DEPh and glycerol was made by dissolving each substance with methanol, by an endothermic process. During the droplet formation and trapping process, the methanol quickly evaporated, and only the two immiscible liquids DEPh and glycerol were left within the droplet. As observed the mixture quickly separated with the DEPh migrating to the surface forming a core-shell microstructure with glycerol left in the core of the droplet. After this separation the DEPh in the shell of the droplet evaporated followed by the glycerol core.

\section{CONCLUSION}

In summary, a new technique that can measure the temporal position-resolved Raman spectra from each $200 \mathrm{~nm} \times 200 \mathrm{~nm}$ area within a laser-trapped single airborne particle was demonstrated. The single particle was trapped using two focused counter-propagating hollow beams. The very stable trapping enables us to measure the slice to slice position-resolved Raman spectra from the whole particle. This spatially resolved single particle Raman technique could also be used to monitor various chemical reactions at different parts of a trapped chemical or biological particle exposed to various environments of interest in life sciences and atmospheric sciences.

\section{ACKNOWLEDGMENT}

Thanks to The Defense Threat Reduction Agency (HDTRA1621520) and the US Army Research Laboratory Mission funds.

\section{REFERENCES}

[1] Redding, B., Schwab, M. \& Pan, Y.L., Raman spectroscopy of optically trapped single biological micro-particles. Sensors, 15(8), pp. 19021-19046, 2015.

[2] Kong, L.B., Zhang, P.F., Wang, G.W., Setlow, P. \& Li, Y.Q., Characterization of Bacterial Spore Germination Using Phase Contrast Microscopy, Raman Spectroscopy and Optical Tweezers. Nat. Protoc., 6, pp. 625-639, 2011.

[3] Dennis-Smither, B.J., Marshall, F.H., Miles, R.E., Preston, T.C. \& Reid, J.P., Volatility and Oxidative Aging of Aqueous Maleic Acid Aerosol Droplets and the Dependence on Relative Humidity. J. Phys. Chem. A, 118(30), pp. 5680-5691, 2014.

[4] Pan, Y.L., Hill, S.C. \& Coleman, M., Photophoretic trapping of absorbing particles in air and measurement of their single-particle Raman spectra. Opt. Express., 20(5), pp. 5325-5334, 2012. 
[5] Kalume, A., Beresnev, L.A., Santarpia, J.L. \& Pan Y.L., Detection and characterization of chemical aerosol using laser-trapping single-particle Raman spectroscopy. Appl. Opt., 56(23), pp. 6577-6582, 2017.

[6] Owen, J.F., Chang R.K. \& Barber, P.W., Morphology-dependent resonances in Raman scattering, fluorescence emission and elastic scattering from microparticles. Aerosol Sci. Tech., 1(3), pp. 293-302, 1982. 